\title{
Exemple ethnographique de l'usage pluri-fonctionnel d'un galet de quartz
}

\author{
par Sophie A. de Beaune
}

Il n'est pas de site paléolithique qui n'ait livré, parmi ses vestiges, des galets ou fragments de galets de pierre dure portant diverses traces d'utilisation. Le cas le plus fréquent est celui du galet présentant des traces de percussion sur une extrémité ou sur les deux et qu'on range en général dans la catégorie des percuteurs. Cette interprétation paraît justifiée par les données expérimentales réunies par les tailleurs de pierre modernes. Cependant, il n'est pas exclu que les mêmes stigmates puissent être obtenus par percussion lancée sur des matières premières dures autres que la pierre.

Il arrive aussi que ces galets portent des traces de percussion sur leurs faces ou sur des arêtes longitudinales; ou encore des traces d'usure par frottement ou abrasion sur leurs extrémités, leurs faces ou leurs arêtes. L'origine de ces traces est alors plus difficile à reconnaître et il ne semble pas s'agir de stigmates liés à la taille du silex. On peut envisager alors toutes sortes d'activités en percussion lancée pour les traces de piquetage, en percussion posée pour les traces d'usure. Enfin, l'aspect luisant ou lustré parfois visible sur les faces usées est généralement attribué au contact avec un solide souple tel que la peau ou le cuir.

Nous-mêmes avons tenté ailleurs une classification typologique de ces galets sur laquelle nous ne reviendrons pas ici (S. de Beaune, 1989). Nous nous arrêterons au fait que, très souvent, ces galets portent plusieurs types de traces qui parfois même se superposent. Il s'agit donc de galets ayant eu plusieurs usages, simultanés ou non.

Un séjour dans un campement touareg de la région d'Agadez au Niger nous a récemment permis d'observer les différentes utilisations que l'on peut faire d'un même galet de quartz (1). Il nous a paru intéressant, pour un préhistorien, d'observer à quels types d'activité ce galet était associé et avec quels gestes il était manipulé. De plus, la propriétaire du galet voyant l'intérêt que nous y portions, a bien voulu nous le donner, ce qui permet de le présenter ici et de souligner sa similitude avec certains galets trouvés en contexte paléolithique.

Ce galet de quartz de forme oblongue mesure $89,5 \mathrm{~mm}$ de longueur et $62,1 \mathrm{~mm}$ de largeur maximale. Son épaisseur atteint $45,5 \mathrm{~mm}$ (fig. 1). On peut considérer qu'il a grossièrement une section triangulaire ; l'une des faces, la plus étendue, est planoconvexe (c) ; les deux autres, qui lui sont opposées ne sont que les deux facettes, séparées par une arête, d'une seule face fortement convexe (a). Ce galet présente des traces de piquetage et d'écrasements sur ses deux extrémités ( $\mathrm{x}$ et $\mathrm{y}$ ), le long de ses arêtes longitudinales (b et $\mathrm{d}$ ) et sur la face plano-convexe où elles sont surtout localisées au centre (c). Par ailleurs, les deux facettes de la face convexe opposée ont été produites par usure (a). De plus, le galet est maculé de nombreuses traces noires : charbon à l'une des extrémités, colorant sur une des facettes lissées par usure et sur l'autre extrémité, enfin traces de pulpe de fruit de Balanites aegyptiaca sur la face plano-convexe (fig. 2).

(1) Il s'agit d'un campement de «forgerons » rattachés aux Touaregs Kel Ferwan situé dans la région d'Agadez, Niger. Le mot de "forgeron » étant celui habituellement utilisé par les ethnologues pour traduire le mot touareg énad, nous l'avons conservé, mais il désigne en fait des artisans aux multiples spécialités. Les forgerons, comme dans de nombreuses populations d'Afrique, forment une classe particulière, de statut ambigu, puisqu'ils sont tout à la fois craints et méprisés. Sur les Touaregs Kel Ferwan et sur les forgerons, je renvoie le lecteur à D. Casajus (1987 a et b). 

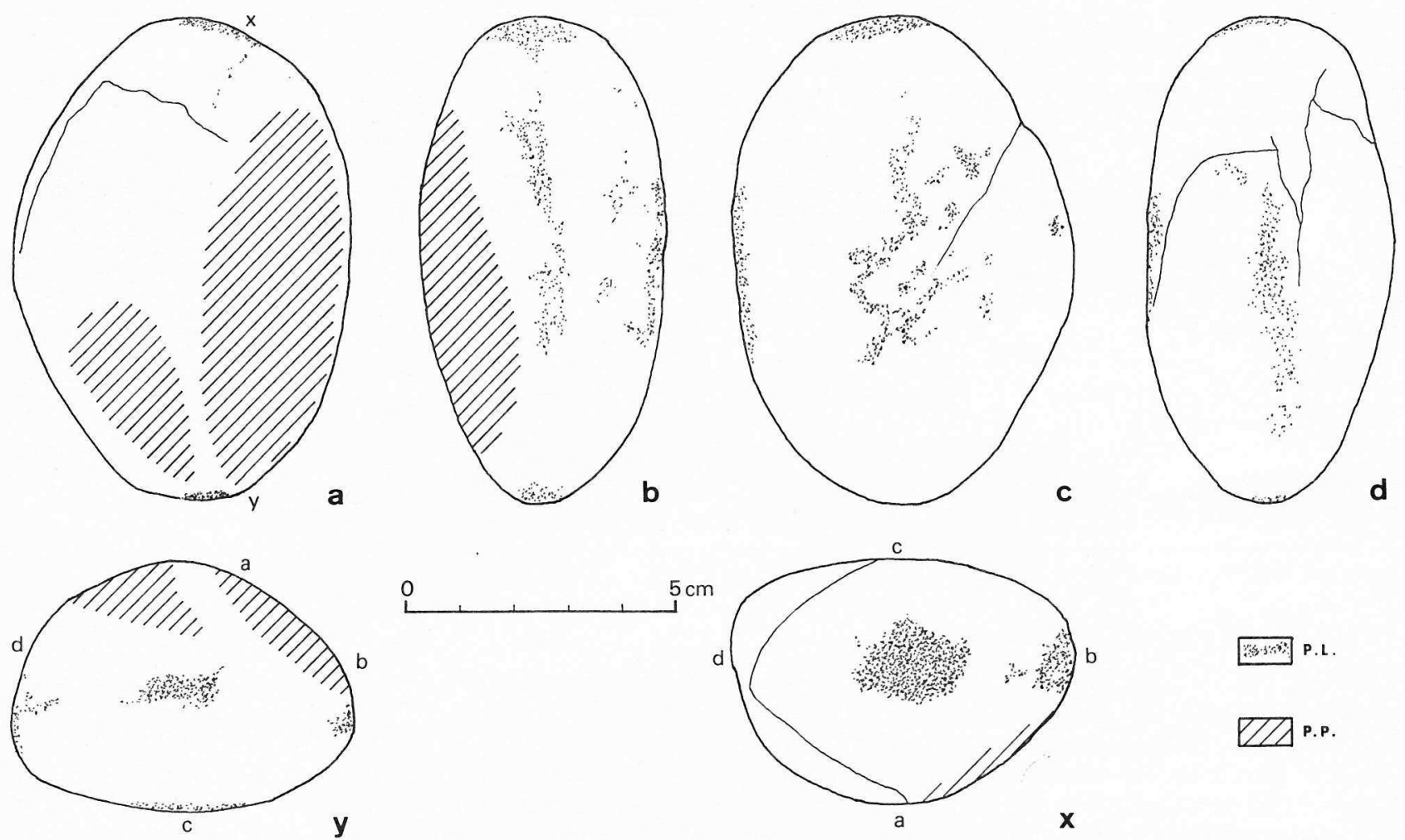

Fig. 1 - Galet de quartz utilisé dans un campement touareg. P.L., percussion lancée ; P.P., percussion posée (dessins S. A. de Beaune)
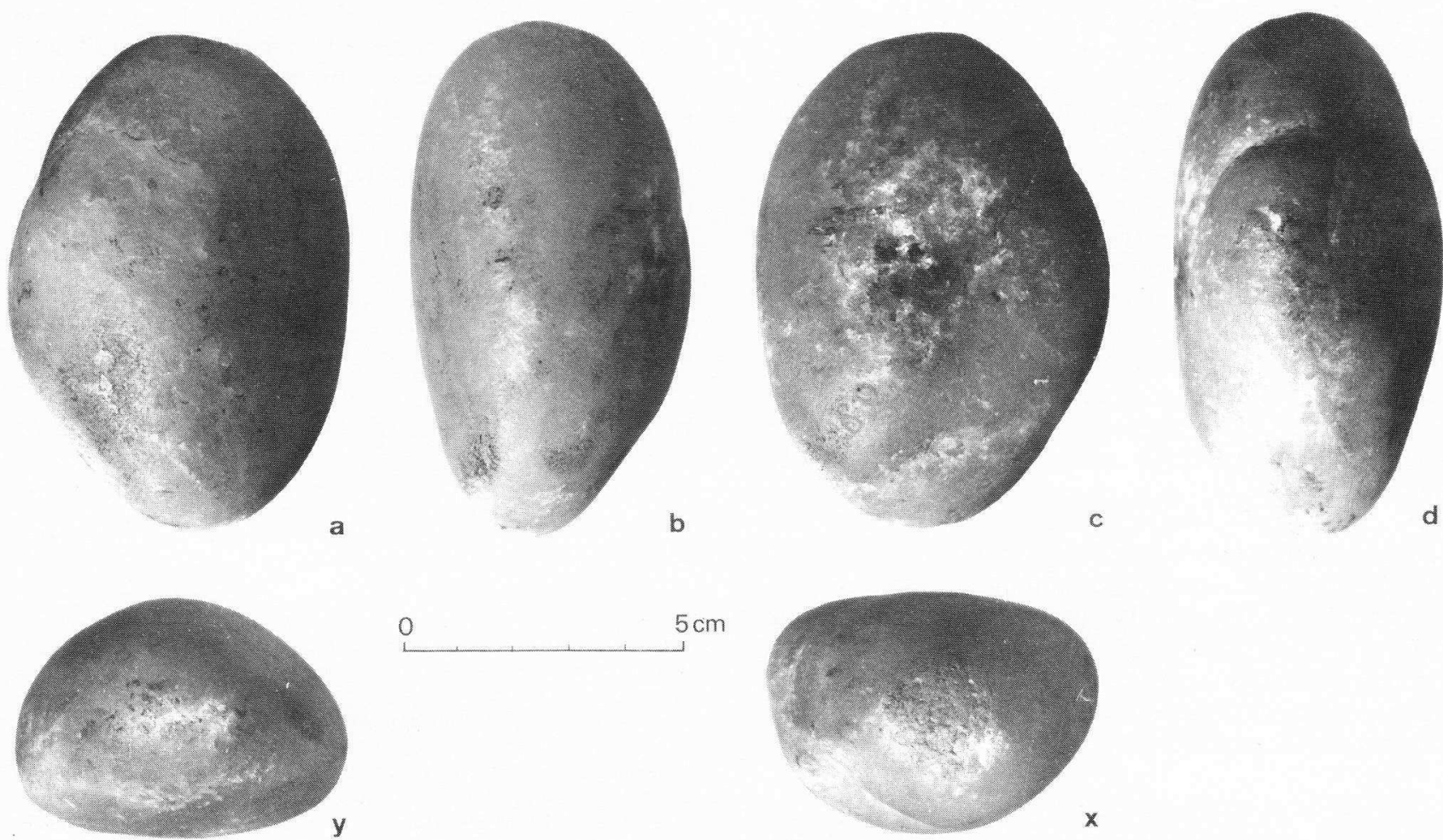

Fig. 2 - Galet de quartz utilisé dans un campement touareg (clichés S. A. de Beaune) 


\section{OBSERVATION ETHNOGRAPHIQUE}

Ce galet que la propriétaire, Akammaya, appelle tablalt, terme générique pour les galets ou simples cailloux, semble utilisé avant tout au lissage (eseslef) du cuir. C'est du moins ainsi que nous le voyons utilisé la première fois (fig. 3). Cet usage prolongé a entraîné la formation des deux grandes facettes d'usure qui portent des traces de colorants employés dans le travail du cuir (indigo ou colorant non identifié appelé localement khorinjan). Quelques jours plus tard, le galet réapparaît dans les mains de Juwa, la mère d'Akammaya, qui l'utilise cette fois comme socle ou support (fig. 4), pour écraser la pulpe d'un akaka, fruit de l'aboragh (Balanites aegyptiaca). Le fruit étant oléagineux, le produit de ce broyage, effectué avec les doigts, est une crème noire dont elle enduit le pied d'un enfant qui souffre d'une brûlure. L'opération d'écrasement a été effectuée sur la face plano-convexe et la pulpe du fruit s'est bien conservée, notamment dans les anfractuosités dues au piquetage antérieur.

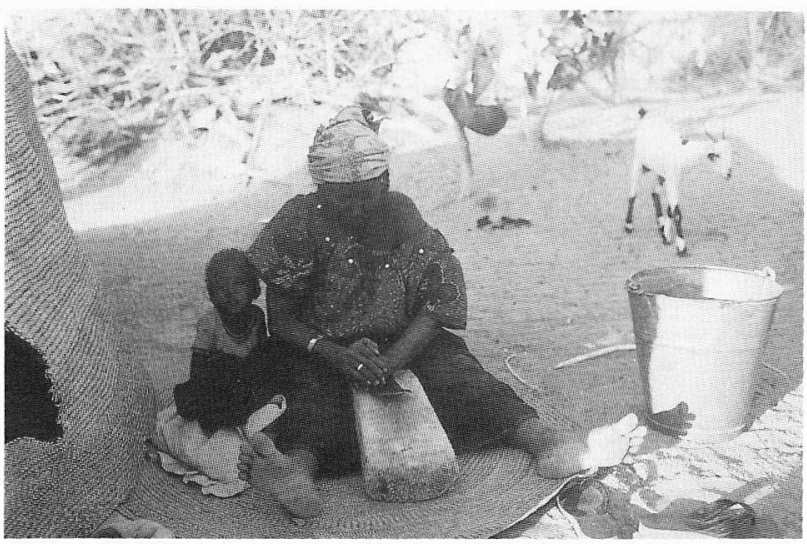

Fig. 3 - Akammaya utilisant le galet sur ses faces longitudinales pour lisser du cuir (cliché S. A. de Beaune).

Après l'avoir vu utilisé comme lissoir puis comme support, j'ai interrogé la propriétaire du galet pour en connaître les autres usages que les traces de piquetage visibles aux extrémités, sur les surfaces et les arêtes permettaient de supposer (2). En ce qui concerne les traces de percussion visibles sur les extrémités, Akammaya explique d'abord qu'elles sont apparues lors du bris de pains de sucre et du broyage de diverses herbes médicinales (amagal) ; dans ce dernier cas, on utilise généralement comme

(2) Je tiens à remercier ici Dominique Casajus qui m'a introduite dans ce campement auquel je n'aurais pu avoir accès sans son aide, et qui m'a servi d'interprète à cette occasion.

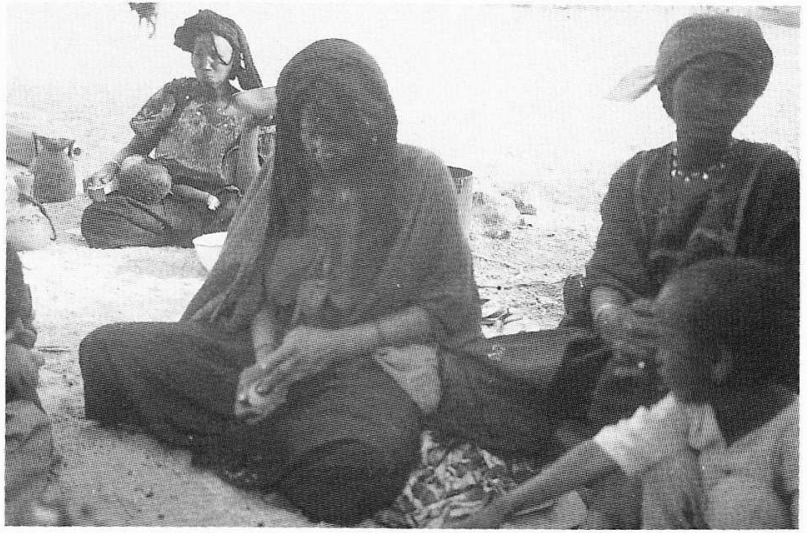

Fig. 4 - Juwa utilisant le galet comme support pour y écraser la pulpe de l'abaka, fruit de l'aboragh (cliché S. A. de Beaune).

surface passive un mortier de bois (tendé) retourné. Interrogée sur les traces de percussion visibles sur la plus grande face, Akammaya commence par les attribuer aux enfants qui s'amusent parfois avec sa pierre. Mais elle ajoute peu après qu'elle utilise parfois le galet sur cette face comme marteau pour repousser du cuir, un burin de fer servant d'intermédiaire.

Nous avons, quelques jours plus tard, observé le mari d'Akammaya, Alkhassan, utilisant la pierre sur un mortier retourné pour broyer du charbon de bois (3). Il tient le galet à deux mains et lui imprime, en plus d'un léger martèlement, un mouvement d'avant en arrière (fig. 5). Il signale qu'on peut de la même manière utiliser ce galet pour broyer l'antimoine devant servir de fard (tazot, khôl).

Akammaya, qui possède deux pierres de ce type, toutes deux en quartz, précise que les Touaregs ne se séparent jamais de ces objets, qu'ils utilisent pour divers tâches de meulage (antimoine, herbes médicinales) (4). Il existe aussi des meules correspondantes mais elle n'en possède pas. La surface du mortier

(3) Alkhassan fabrique des bijoux d'argent et utilise le charbon broyé pour noircir certains traits gravés sur la surface des bijoux. Le geste qu'il effectue est rendu par le verbe adäbdäb, idéophone pouvant être traduit par « marteler, donner de petits coups ».

(4) Ce galet ayant été observé chez des forgerons, certains de ces usages leur sont spécifiques (travail du cuir, broyage du charbon de bois). Mais l'utilisation à laquelle Akammaya fait ici allusion est susceptible d'être observée chez d'autres Touaregs. Par ailleurs, D. Casajus m'a rapporté que d'autres Touaregs, non forgerons, possédaient également des galets de ce type mais pouvant avoir un autre usage. Dans un cas qu'il a observé, il s'agissait d'un galet utilisé sur ses faces pour donner leur aspect définitif aux cordes tressées faites de palmes de doum (tagäyt, Hyphaene thebaïca) destinées à entraver les chameaux. La corde est martelée à l'aide du galet, une autre pierre, plus volumineuse et à surface convexe, servant d'enclume. 


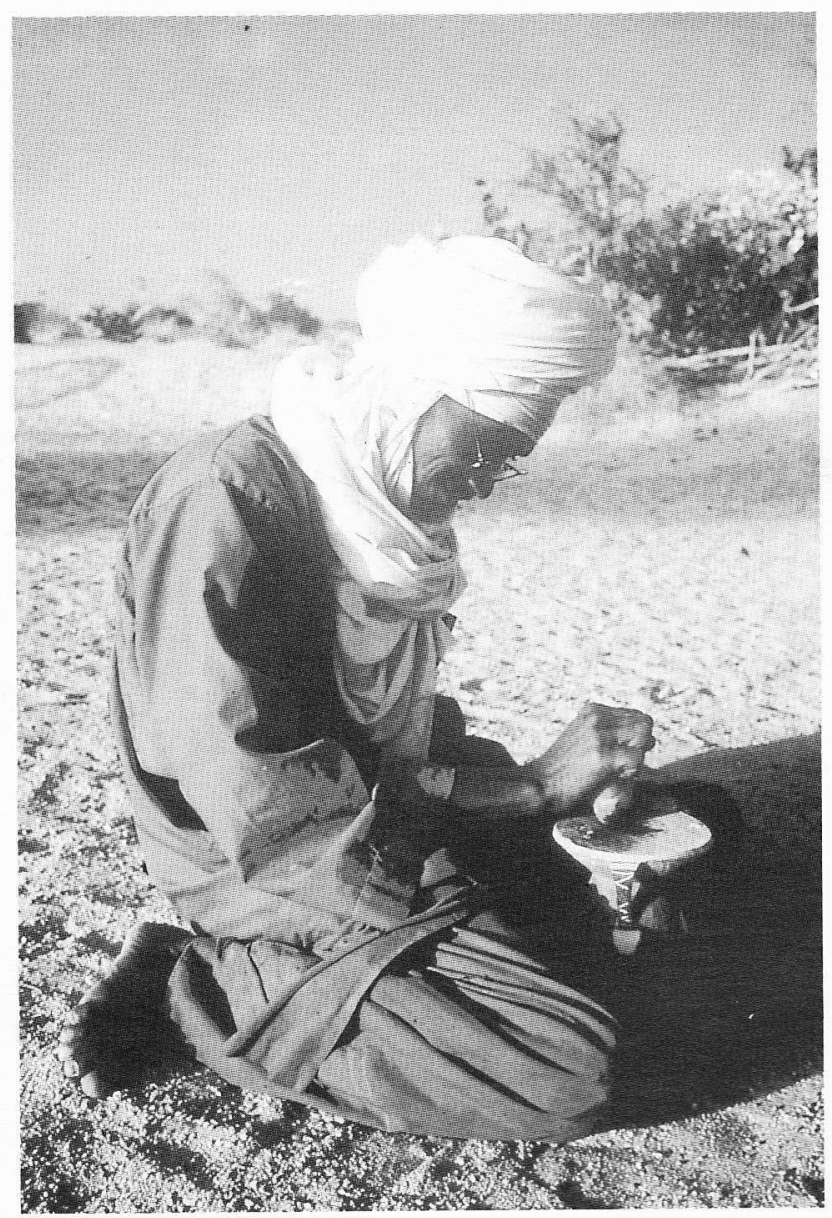

Fig. 5 - Alkhassan broyant du charbon de bois sur le mortier retourné à l'aide du même galet (cliché $S$. A. de Beaune).

retourné, circulaire et plane ou légèrement concave, correspond du reste bien à la forme et aux dimensions d'une meule. Quant au support utilisé pour le lissage des cuirs, il s'agit d'un grand socle de bois (telkäläb) qui sert également à toutes sortes d'usage passif et même, à l'occasion, d'oreiller ou de petit siège.

On peut associer les divers usages de ce galet avec les traces d'utilisation obtenues dans le tableau suivant :

\begin{tabular}{|l|l|l|}
\hline \multicolumn{1}{|c|}{ USAGE } & TRACES D'UTILISATION \\
\hline ACTIF & $\begin{array}{l}\text { Percuteur (sucre en pain) } \\
\text { Broyeur (herbes médici- } \\
\text { nales, charbon) } \\
\text { cuir) } \\
\text { Jouets d'enfants ? }\end{array}$ & $\begin{array}{l}\text { Traces de percussion aux } \\
\text { extrémités } \\
\text { Traces de percussion aux } \\
\text { extrémités et sur arêtes et } \\
\text { coloration brune } \\
\text { Traces de percussion sur } \\
\text { surfaces } \\
\text { Traces de percussion aux } \\
\text { extrémités, sur arêtes et } \\
\text { surfaces } \\
\text { Création de facettes usées } \\
\text { et luisantes sur surfaces et } \\
\text { coloration }\end{array}$ \\
\hline PASSIF & $\begin{array}{l}\text { Support (broyage de } \\
\text { pulpe de fruit) }\end{array}$ & $\begin{array}{l}\text { Coloration et imprégna- } \\
\text { tion de la pulpe dans la } \\
\text { roche }\end{array}$ \\
\hline
\end{tabular}

Le caractère pluri-fonctionnel de ce galet nous paraît très révélateur et nous avions déjà souligné cette caractéristique pour certains ustensiles utilisés par les Aborigènes australiens (S. de Beaune, 1989). Si la classification typologique des galets utilisés au Paléolithique que nous avons présentée ailleurs reste pour l'instant valide dans la théorie, il ne faut pas oublier que nous pouvons avoir affaire, dans un grand nombre de cas, à des objets composites. De même que, pour les spécialistes du silex, il existe des grattoirs-burins, de même on peut envisager ici l'existence de broyeurs-lissoirs, de percuteurs-enclumes... De plus, si ces galets ont probablement eu plusieurs usages comme nous venons de le montrer, leur caractère passif ou actif ne devait pas être aussi marqué qu'il peut le sembler dans la théorie. En effet, pour des objets de faibles dimensions, comme c'est le cas-ici, qui présentent donc un caractère mobile et portatif, on peut envisager un usage actif mobile (en percuteur ou lissoir par exemple) et un usage passif, l'objet étant maintenu immobile dans le creux de la main, comme ici pour le broyage de la pulpe de l'abaka.

Du reste, nous avons déjà pu observer de nombreux exemples paléolithiques d'outils pluri-fonctionnels, notamment dans la remarquable collection de R. de Saint-Périer provenant de la grotte d'Isturitz. Rappelons que des molettes d'Isturitz ont été réutilisées comme enclumes au centre de leurs faces inférieure et supérieure (S. de Beaune, 1989). Elles ont donc eu un usage actif, puis passif, ce qui rappelle certains types d'enclumes (kulki) connues en Australie. On voit donc que le caractère pluri-fonctionnel des ustensiles paléolithiques augmente la difficulté d'interprétation de leurs traces d'usage et que le recours à des données d'ordre ethnographique peut apporter un complément d'informations non négligeable ou au moins un faisceau d'hypothèses originales.

Sophie A. de BEAUNE

U.A. 275 du C.N.R.S.

44, rue de l'Amiral-Mouchez 75013 PARIS

BeAunE S. A. de (1987) - Communication. Esquisse d'une classification des galets et plaquettes utilisés au Paléolithique. Bulletin de la Société préhistorique française, t. 84, n 7 , pp. 197198, 1 tabl.

Beaune S. A. de (1989) - Essai d'une classification typologique des galets et plaquettes utilisés au Paléolithique. Gallia Préhistoire, vol. 31, 35 p., 14 fig., 3 tabl.

Casajus D. (1987 a) - La tente dans la solitude. La société et les morts chez les touaregs Kel Ferwan. Cambridge University Press. Cambridge. Éditions de la Maison des Sciences de l'Homme. Paris, 390 p., 41 fig.

Casajus D. (1987 b) - Crafts and ceremonies : the inadan in tuareg society in: Aparna Rao (éd.) The other Nomads; Peripatetic minorities in cross-cultural perspective. Böhlau Verlag. Köln, Wien, pp. 291-310. 